\title{
AUF1/hnRNP D is a novel protein partner of the EBER1 noncoding RNA of Epstein-Barr virus
}

\author{
NARA LEE, GENARO PIMIENTA, and JOAN A. STEITZ1 \\ Department of Molecular Biophysics and Biochemistry, Howard Hughes Medical Institute, Yale University School of Medicine, \\ New Haven, Connecticut 06536, USA
}

\begin{abstract}
Epstein-Barr virus (EBV)-infected cells express two noncoding RNAs called EBV-encoded RNA (EBER) 1 and EBER2. Despite their high abundance in the nucleus (about $10^{6}$ copies), the molecular function of these noncoding RNAs has remained elusive. Here, we report that the insertion into EBER1 of an RNA aptamer that binds the bacteriophage MS2 coat protein allows the isolation of EBER1 and associated protein partners. By combining MS2-mediated selection with stable isotope labeling of amino acids in cell culture (SILAC) and analysis by mass spectrometry, we identified AUF1 (AU-rich element binding factor 1)/hnRNP D (heterogeneous nuclear ribonucleoprotein D) as an interacting protein of EBER1. AUF1 exists as four isoforms generated by alternative splicing and is best known for its role in destabilizing mRNAs upon binding to AU-rich elements (AREs) in their $3^{\prime}$ untranslated region (UTR). Using UV crosslinking, we demonstrate that predominantly the p40 isoform of AUF1 interacts with EBER1 in vivo. Electrophoretic mobility shift assays show that EBER1 can compete for the binding of the AUF1 p40 isoform to ARE-containing RNA. Given the high abundance of EBER1 in EBV-positive cells, EBER1 may disturb the normal homeostasis between AUF1 and ARE-containing mRNAs or compete with other AUF1-interacting targets in cells latently infected by EBV.
\end{abstract}

Keywords: AUF1; EBER1; noncoding RNA

\section{INTRODUCTION}

Epstein-Barr virus (EBV) is a human $\gamma$-herpesvirus that is associated with B-cell malignancies, such as Burkitt's and Hodgkin's lymphomas, as well as with several epithelial cancers, including nasopharyngeal and gastric carcinomas (Young and Rickinson 2004). Encoded in the EBV genome are two evolutionary conserved noncoding RNAs called EBV-encoded RNA (EBER) 1 and EBER2, which are nonpolyadenylated, RNA polymerase III transcripts of 167 and 172 nucleotides (nt), respectively. EBERs accumulate to over $10^{6}$ copies in the nucleus during all stages of latent infection (Lerner et al. 1981; Rosa et al. 1981; Glickman et al. 1988).

Although the molecular mode of action of EBERs is not understood, phenotypic observations point to a role in EBV oncogenicity. The expression of EBERs in B lymphocytes results in colony formation in soft agar and tumor induction in nude mice (Komano et al. 1999; Ruf et al. 2000). Recent reports have shown that EBER1 alone is sufficient to elicit these phenotypes (Houmani et al. 2009; Repellin et al.

\footnotetext{
${ }^{1}$ Corresponding author

E-mail joan.steitz@yale.edu

Article published online ahead of print. Article and publication date are at http://www.rnajournal.org/cgi/doi/10.1261/rna.034900.112.
}

2010). Moreover, EBERs have been suggested to enhance growth transformation efficiency in EBV-infected B lymphocytes (Yajima et al. 2005). This point remains controversial, however, as other studies have concluded that EBERs do not affect EBV-induced growth transformation (Swaminathan et al. 1991; Gregorovic et al. 2011). Transcription profile changes that contribute to tumor formation after expression of EBERs have also been reported, such as up-regulation of various cytokines depending on the cell type: interleukin-10 in B lymphocytes, interleukin-9 in T cells, and insulin-like growth factor 1 in nasopharyngeal carcinoma cells (Kitagawa et al. 2000; Iwakiri et al. 2003; Yang et al. 2004).

Originally, EBERs were discovered to form stable ribonucleoprotein (RNP) complexes with the cellular protein La (Lerner et al. 1981), which binds all nascent RNA polymerase III transcripts and aids in their maturation (Wolin and Cedervall 2002). The large ribosomal subunit protein L22 was subsequently identified as another partner of EBER1 (Toczyski and Steitz 1991; Toczyski et al. 1994). No specific ligands for EBER2 have yet been identified.

Here, we aimed to provide further insight into the molecular mode of action of the EBER1 RNP by identifying novel RNA-protein interactions. We selected EBER1 RNPs from cell extracts using complementary oligonucleotides and 
RNA aptamers, which are nucleotide sequences with strong affinity for specific molecules (Srisawat and Engelke 2002; Zhou et al. 2002). We show that insertion of a widely used RNA aptamer for the bacteriophage MS2 coat protein enables the isolation of EBER1 and associated proteins. Combining this aptamer-mediated selection with mass spectrometry analysis, we identified AU-rich element binding factor 1 (AUF1)/heterogeneous nuclear RNP D (hnRNP D) as a novel interacting protein of EBER1 both in vitro and in vivo. AUF1 is best known for its ability to bind AU-rich elements (AREs), which commonly reside in $3^{\prime}$ untranslated regions (UTRs), and to enhance the decay of many shortlived mRNAs, including those of proto-oncogenes, cell cycle regulators, and cytokines (Caput et al. 1986; Chen and Shyu 1995; Loflin et al. 1999). However, stabilizing effects of AUF1 have also been reported ( $\mathrm{Xu}$ et al. 2001), as well as diverse functions in aging, telomere maintenance, and suppression of senescence (Pont et al. 2012). AUF1 is expressed as four isoforms ( $\mathrm{p} 37, \mathrm{p} 40, \mathrm{p} 42, \mathrm{p} 45)$ that originate from alternative splicing events and have been ascribed nonredundant functions (Wagner et al. 1998; Arao et al. 2000; Sarkar et al. 2003; Raineri et al. 2004; Kedar et al. 2012). Given the observation that AUF1 overexpression leads to tumorigenesis (Gouble et al. 2002), the interplay between AUF1 and EBER1 could contribute to the oncogenic phenotype of EBER1-expressing cells.

\section{RESULTS}

\section{Selection of EBER1 RNP complexes using an RNA aptamer for the bacteriophage MS2 coat protein}

To identify novel EBER1-interacting proteins, we sought a system that facilitates the direct capture of EBER1 from cell lysates. We used BJAB cells, an EBV-negative Burkitt's lymphoma B-cell line, and BJAB-B1 cells, which are derived from $\mathrm{BJAB}$ cells by in vitro infection with EBV (Menezes et al. 1975; Fresen et al. 1977). First, we investigated complementary oligonucleotides as a tool to select EBER1. To identify accessible region(s) of EBER1, RNase $\mathrm{H}$ cleavage assays were performed by adding seven $\sim 30$-nt-long antisense DNA oligonucleotides to BJAB-B1 cell lysate to induce EBER1 digestion by endogenous RNase H (Supplemental Fig. S1; Wassarman and Steitz 1991). Only the oligonucleotide complementary to stem-loop III of EBER1 induced $\mathrm{RNase} \mathrm{H}$ digestion, indicating that this region is partially available in the RNP. A 2'-O-methyl modified RNA oligonucleotide complementary to stem-loop III was covalently attached to adipic acid dihydrazide Agarose beads (Borah et al. 2009) and incubated with EBV-positive BJABB1 cell lysate (Fig. 1A). Although we were able to select $\sim 5 \%$ of total EBER1 RNA, known interacting proteins of EBER1, such as La and L22, were not coselected (Fig. 1D).

Next, we attempted to select EBER1 by inserting a single S1-aptamer, which has high affinity for streptavidin (Srisawat and Engelke 2002), into several sites in the RNA. Insertion only into stem-loop IV preserved the affinity of S1 for streptavidin, whereas insertions into the $5^{\prime}$ - or $3^{\prime}$-end or into stem-loop III did not (Fig. 1B; data not shown). BJAB cells that express EBER1 containing the S1-aptamer (EBER1-S1) were generated by lentiviral transduction (Cao et al. 2008). Approximately 5\% of total EBER1-S1 RNA could be captured on streptavidin beads from lysate prepared from these cells. However again, the EBER1-interacting proteins La and L22 failed to be coselected (Fig. 1D).

Finally, we inserted three MS2-RNA hairpins, which bind the MS2 bacteriophage coat protein (Zhou et al. 2002), at the $5^{\prime}$ - or $3^{\prime}$-end as well as into stem-loop IV of EBER1, and generated BJAB cells expressing these constructs by lentiviral transduction (Cao et al. 2008). The $\mathrm{BJAB}$ cells expressing EBER1 with MS2-hairpin insertions in stem-loop IV (EBER1-MS2) (Fig. 1C) exhibited a 12fold higher expression level than the other EBER1-MS2 constructs (data not shown). The addition of MS2 coat protein recombinantly expressed in bacteria as a fusion protein with maltose binding protein (MBP) to the lysate with amylose resin recovered up to 20\% of EBER1-MS2 RNA. More importantly, EBER1-interacting proteins La and L22 were coselected (Fig. 1D).

\section{SILAC coupled with mass spectrometry identifies novel protein partners of EBER1}

To investigate proteins purified by EBER1-MS2 selection, we resolved the maltose eluate by SDS-gel electrophoresis and compared the eluted proteins to those from lysate containing unmodified EBER1. Although the silver-stained protein profiles revealed no obvious differences, the known EBER1 interactors La and L22 were specifically enriched only in the EBER1-MS2 containing lysate upon Western blot analysis (Supplemental Fig. S2).

To distinguish interacting proteins from the background of nonspecific contaminants, SILAC (stable isotope labeling of amino acids in cell culture) in combination with mass spectrometry analysis was employed (Blagoev et al. 2003; Mann 2006). In this method, cell lines are cultured in medium containing the essential amino acids arginine and lysine bearing either naturally abundant ${ }^{12} \mathrm{C},{ }^{14} \mathrm{~N}$ (light) or ${ }^{13} \mathrm{C}$, ${ }^{15} \mathrm{~N}$ (heavy) isotopes. After selection, specific binding proteins are identified by mass spectrometry through the relative enrichment of peptides containing the isotope originating from one cell line, whereas contaminating proteins yield light and heavy peptides in equal abundance. EBER1 and EBER1-MS2 expressing cells were cultured in light and heavy medium, respectively, and equal amounts of lysate were mixed prior to MS2-aptamer selection. EBER1-MS2 RNP complexes were eluted from amylose resin by the addition of maltose, and the proteins were size-fractionated by SDS-gel electrophoresis prior to mass spectrometry (Fig. 2A). MaxQuant software was used to determine the SILAC 
A

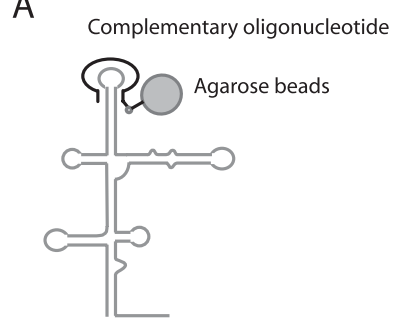

B

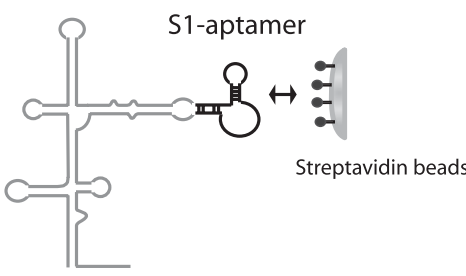

C

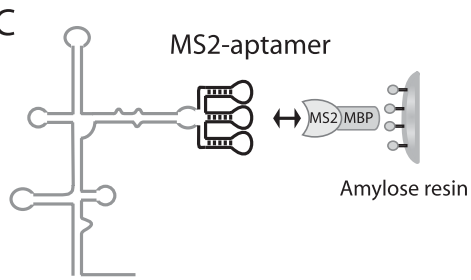

D

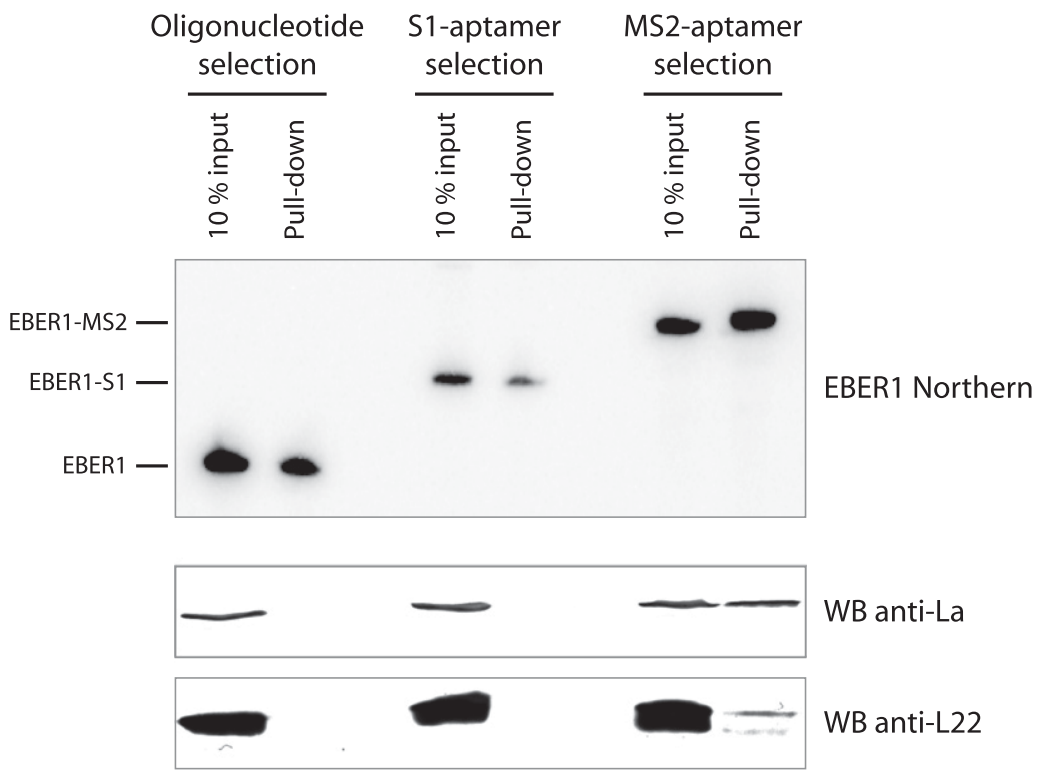

FIGURE 1. The MS2-aptamer selects EBER1-interacting proteins. $(A-C)$ Three strategies for selecting EBER1 RNPs are depicted. (A) EBER1 was isolated using a complementary oligonucleotide covalently linked to adipic acid dihydrazide Agarose beads. Alternatively, an aptamer was inserted into stem-loop IV of EBER1. (B) The S1-aptamer (45 nt) or (C) three MS2-hairpin loops (82 nt) were used to select EBER1 RNPs on streptavidin beads or on MS2 coat-MBP fusion protein tethered to amylose resin, respectively. $(D)$ For selection with complementary oligonucleotides, BJABB1 cell lysate was used. For aptamer-mediated selections, stable BJAB cell lines were generated that express either EBER1-S1 or EBER1-MS2. A Northern blot probed for EBER1 assessed the pull-down efficiency of aptamer-containing EBER1 RNAs versus unmodified EBER1. Western blots using antibodies against La and L22 asked whether known EBER1-interacting proteins were coselected.

ratio of eluted proteins, thus identifying candidate interacting proteins (Supplemental Table S1). Among the proteins that displayed SILAC ratios characteristic of specific interactors were the previously established EBER1-interacting proteins La and L22, as well as AUF1 (Supplemental Fig. S3).

We confirmed AUF1 as an EBER1-interacting protein using anti-AUF1 antibodies (Pinol-Roma et al. 1988). There are four AUF1 spliced isoforms: All contain two RNA recognition motifs and a C-terminal glutamine-rich domain, but differ by the presence or absence of two regions called inserts A and B (Fig. 2B). MS2-aptamer selection predominantly captured the $\mathrm{p} 40$ and/or $\mathrm{p} 42$ isoform of AUF1, which comigrates in the gel, and, more weakly, the p45 isoform (Fig. 2C). The p37 isoform was not selected. Lysate containing unmodified EBER1 provided a negative control.

\section{EBER1 predominantly interacts with the $\mathrm{p} 40$ isoform of AUF1 in vivo}

To demonstrate that the interaction of AUF1 with EBER1 occurs in vivo, we used a previously developed method that entails UV crosslinking of intact cells, immunoprecipitation, and washing under stringent conditions (Cook et al.
2004). The AUF1 peptide sequence identified by mass spectrometry analysis could not discriminate between the four isoforms, as it lies within the RNA recognition motif II common to all isoforms (Fig. 2B). We therefore individually cotransfected into HEK293T cells plasmids expressing a Flag-tagged version of each AUF1 isoform as well as an EBER1 plasmid, UV-irradiated and coimmunoprecipitated crosslinked EBER1 using anti-Flag antibodies (Fig. 3A). While all isoforms of AUF1 detectably bound EBER1, compared to coprecipitation in the absence of UV crosslinking, the p40 isoform showed the most pronounced interaction (Fig. 3B). To distinguish between preferential binding of the p40 AUF1 isoform to EBER1 and enhanced crosslinking efficiency of this isoform, we repeated the immunoprecipitation of Flag-tagged $\mathrm{p} 40^{\mathrm{AUF1}}$ in the presence of Myc-tagged p40 and p45 isoforms. If the p40 isoform is preferentially bound to EBER1, only the Myc-tagged p40 isoform should interfere with EBER1 coimmunoprecipitation by anti-Flag antibodies. Indeed, Figure 3C shows a decrease in EBER1 coimmunoprecipitation in the presence of Myc-tagged $\mathrm{p} 40^{\mathrm{AUF1}}$ compared with coexpression of Myc-tagged $\mathrm{p} 45^{\mathrm{AUF1}}$, arguing that the p40 isoform preferentially binds EBER1.

An in vivo interaction of the p40 isoform and EBER1 is supported by the fact that both molecules are found in the 
A

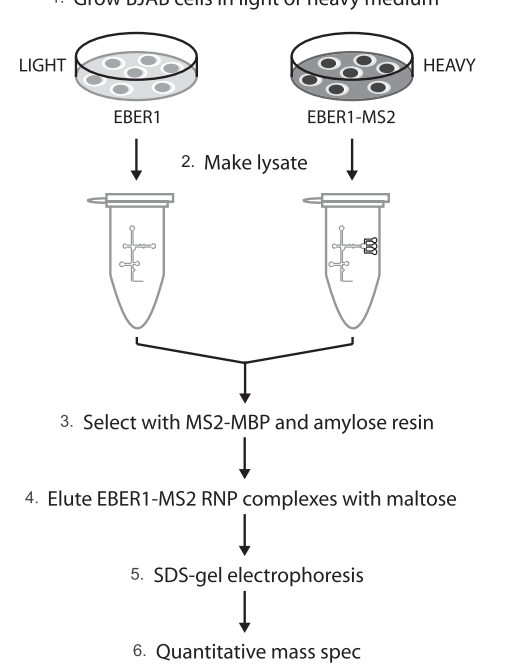

B

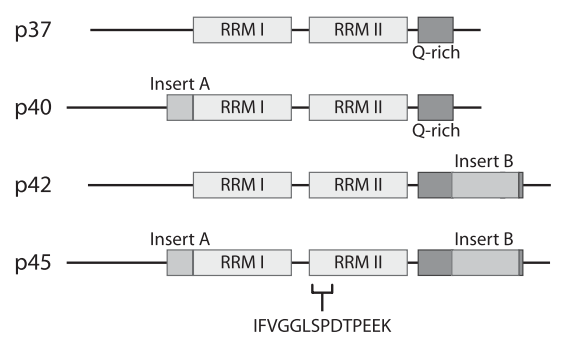

C
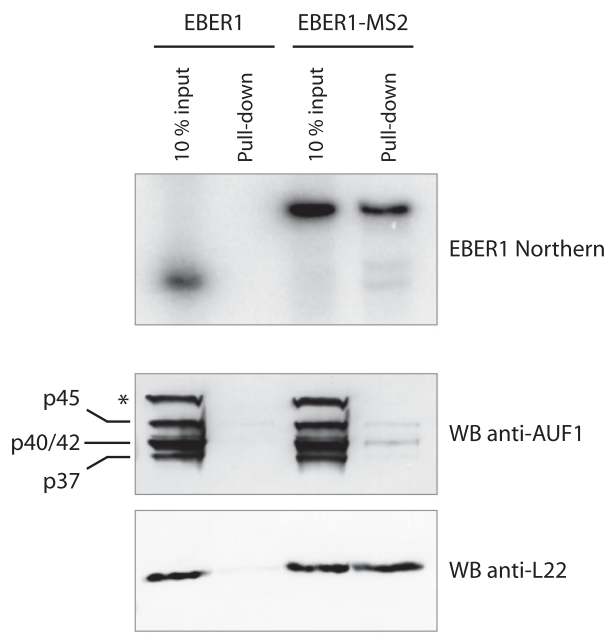

FIGURE 2. AUF1/hnRNP D is a novel EBER1-interacting protein. (A) Experimental outline of the EBER1-MS2 selection coupled to SILAC and mass spectrometry analysis. (B) Schematics of the four isoforms of AUF1 (Wagner et al. 1998), which originate from alternative splicing and differ by the presence or absence of two regions called Insert A and Insert B. The peptide sequenced by tandem mass spectrometry analysis is part of the RNA recognition motif (RRM) II domain as indicated at the bottom. Q-rich indicates glutamine-rich domain. (C) Confirmation of the specificity of the EBER1-MS2 selection of AUF1. Lysates of BJAB cells containing either EBER1 or EBER1-MS2 were subjected to MS2-mediated selection. RNA was isolated and probed for EBER1 by Northern blotting to determine the pull-down efficiency. Western blots for AUF1 showed that AUF1 is selected only from cell lysates containing EBER1-MS2 and not from lysates containing unmodified EBER1. The four isoforms of AUF1 are indicated; the p40 and p42 isoforms comigrate in the gel. The asterisk indicates a cross-reactive band. Western blot analysis of L22 is shown as a positive control.

same subcellular compartment (the p40 isoform localizes to both cytoplasm and nucleus [Xu et al. 2001], whereas EBER1 is strictly nuclear [Fok et al. 2006a]). To confirm the in vivo interaction of endogenous AUF1 and EBER1 in EBV-positive B lymphocytes, we used Raji cells, which are Burkitt's lymphoma cells naturally infected with EBV (Drexler and Minowada 1998), and antibodies against AUF1 (Dempsey et al. 1998) as well as L22, as a positive control for in vivo UV crosslinking and subsequent immunoprecipitation. EBER1 was coimmunoprecipitated only upon UV irradiation with anti-AUF1 and, as expected, anti-L22 antibodies (Fig. 3D, upper panel). We probed the same Northern blot for EBER2 as a specificity control, and observed no binding for either protein (Fig. 3D, lower panel). We conclude that EBER1 interacts in vivo with the p40 isoform of AUF1.

\section{EBER1 can compete with the binding of AUF1 to ARE RNA in vitro}

Since AUF1 binds the 3' UTRs of ARE-containing mRNAs to regulate their stability (Chen and Shyu 1995; Loflin et al. 1999), we asked whether AUF1 interaction might affect the level of EBER1 in EBV-infected cells. We generated BJABB1 cells that express a doxycycline-inducible short hairpin RNA that reduces the amounts of all isoforms of AUF1 protein to $\sim 10 \%$ of the endogenous level (Supplemental Fig. S4A). Despite this marked depletion of AUF1, EBER1 levels in EBV-infected cells remained unaltered $5 \mathrm{~d}$ post doxycycline treatment, suggesting that AUF1 does not affect the stability of EBER1 (Supplemental Fig. S4B).

We also examined the possibility that EBER1 interferes with the interaction between AUF1 and ARE-containing mRNAs. We in vitro transcribed 38 nt of the core ARE from the TNF $\alpha$-mRNA as well as EBER1 and performed electrophoretic mobility shift assays with recombinant $\mathrm{p} 40^{\mathrm{AUF}}$ protein (see Materials and Methods). The TNF $\alpha-$ ARE formed a single RNP complex with $\mathrm{p} 40^{\mathrm{AUF} 1}$, as previously described (Fig. 4A; Zucconi et al. 2010). EBER1 formed four distinct RNP complexes with $\mathrm{p} 40^{\mathrm{AUF}}$, suggesting that multiple regions of EBER1 interact with AUF1 (Fig. 4B). This behavior is reminiscent of RNP complex formation between EBER1 and L22, in which multiple stem-loops within EBER1 contribute to the RNA-protein interaction (Fok et al. 2006b). AUF1 and L22, however, form distinctly different RNP complexes with EBER1, since single stem-loop deletions did not result in a decrease in the overall number of AUF1-EBER1 RNP complexes (data not shown), in contrast to L22 (Fok et al. 2006b). EBER1 and the TNF $\alpha$-ARE formed RNP complexes at comparable AUF1 concentrations (Fig. 4, cf. A and B). Accordingly, we observed that a 2.5-fold molar excess of EBER1 effectively competes with the ARE for binding to $\mathrm{p} 40^{\mathrm{AUF} 1}$ in bandshift assays (Fig. 4C). To corroborate the specificity of EBER1 in this competition, we used the adenoviral VAI RNA, another viral noncoding RNA of similar length $(\sim 160 \mathrm{nt})$ transcribed by RNA polymerase III (Steitz et al. 2011), and 
A

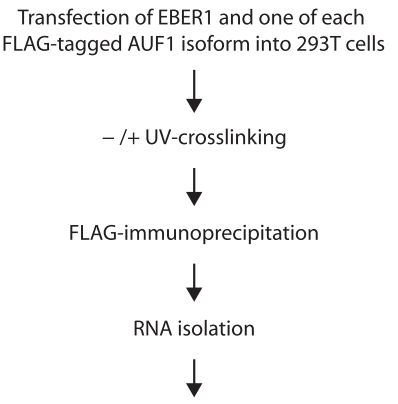

EBER1 detection by Northern blot

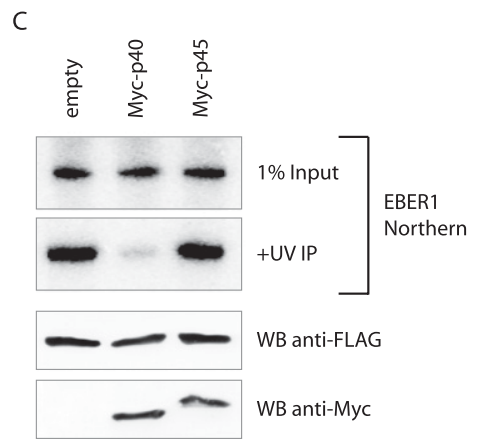

B

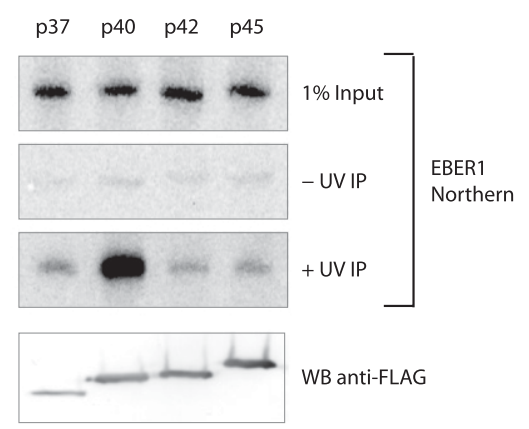

D

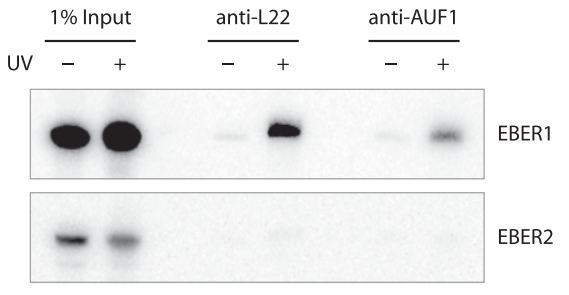

FIGURE 3. EBER1 binds predominantly the p40 isoform of AUF1 in vivo. $(A)$ Experimental outline for UV crosslinking and coimmunoprecipitation of EBER1 to detect in vivo interactions. (B) The four Flag-tagged isoforms of AUF1 were transiently expressed individually, along with EBER1, in HEK293T cells. Immunoprecipitation using anti-Flag antibodies was carried out either omitting or including UV crosslinking, followed by RNA isolation and detection of EBER1 by Northern blot. All AUF1 isoforms bind EBER1 in vivo, with the p40 isoform showing the strongest crosslinking efficiency. Anti-Flag antibodies were used in a Western blot to verify the expression of each AUF1 isoform. (C) Competition experiments were carried out, showing that the enhanced interaction of p $40^{\mathrm{AUF} 1}$ with EBER1 is due to preferential binding rather than preferential crosslinking efficiency. Flag-p40 ${ }^{\mathrm{AUF} 1}$ and EBER1 were coexpressed in HEK293T cells with either an empty vector, Myc-tagged p40 AUF1, or Myc-tagged $\mathrm{p} 45^{\mathrm{AUF} 1}$, and immunoprecipitation was carried out using anti-Flag antibodies. The plasmids expressing Myc-tagged AUF1 were transfected in 10-fold molar excess over the Flag-tagged isoform. Western blots using anti-Flag or anti-Myc antibodies were carried out to show comparable expression of Flag-p $40^{\mathrm{AUF} 1}$ or the Myc-tagged AUF1 isoforms. (D) In vivo UV crosslinking of Raji cells was followed by immunoprecipitation using anti-L22 (as a positive control) and anti-AUF1 antibodies, and EBER1 was detected by Northern blot analysis. The same blot was probed for EBER2 as a specificity control.

observed a decrease in ARE binding only at a much higher molar excess (25-fold) (Fig. 4D). Thus, high levels of EBER1 could potentially interfere with AUF1 binding to cellular ARE-containing mRNAs in EBV-transformed cells.

\section{DISCUSSION}

Although several EBER1-interacting proteins have been identified to date, the molecular mode of action and, in particular, the oncogenic influence of the EBER1 RNP remain poorly understood. In an attempt to identify novel interacting partners that might shed light on the molecular function of the EBER1 RNP, we established a method employing the MS2-hairpin aptamer to capture EBER1 RNPs directly from cell lysates. By combining EBER1-MS2 selection with mass spectrometry, AUF1 was identified as a novel interacting partner of EBER1. Preferential binding of the $\mathrm{p} 40$ isoform relative to other AUF1 isoforms in vivo provided evidence of a specific interaction. Our observation that EBER1 can compete for the binding of AUF1 to a classical ARE RNA in electrophoretic mobility shift assays suggests a physiologically relevant function for this interaction.

Many other RNPs have been captured utilizing complementary oligonucleotides (Wassarman and Steitz 1991; Borah et al. 2011; Simon et al. 2011), an approach whose primary advantage is that no modifications to the RNA are required. Surprisingly, although EBER1 could be selected by an antisense oligonucleotide, $\mathrm{La}$ and $\mathrm{L} 22$, the best-characterized EBER1 interacting partners, were not coselected. The region of EBER1 we identified as a target for hybridization and RNase H cleavage overlaps with stemloop III, previously characterized as the major binding site for L22 (Toczyski and Steitz 1993; Fok et al. 2006b). Whereas in prior immunoprecipitation experiments $>95 \%$ of total EBER1 was precipitated by anti-L22 antibodies (Toczyski and Steitz 1993), oligonucleotide selection captured only $5 \%$ (Fig. 1D), suggesting that only EBER1 molecules not associated with L22 are isolated by this method. Similar to selection with complementary oligonucleotides, S1-aptamer-tagged EBER1 was retained on streptavidin beads, but again neither La nor L22 was copurified. Inserting RNA aptamers has the inherent risk of rendering RNAs nonfunctional, particularly in case of noncoding RNAs, where both sequence and structure can be critical for function. It is possible that the addition of nucleotides disrupts the proper assembly of EBER1 with La and L22. However, we were able to coimmunoprecipitate EBER1-S1 using anti-L22 antibodies (data not shown), supporting the existence of two distinct pools of EBER1-S1: one that binds L22 but cannot be isolated by streptavidin, and one that retains affinity for streptavidin but does not bind L22.

Several interacting proteins were identified by EBER1MS2 selection coupled to mass spectrometry. In addition to AUF1, two other hnRNP proteins (hnRNP A1 and hnRNP A2/B1) exhibited SILAC ratios indicating association with EBER1 (Supplemental Table S1). In the case of AUF1, we 
A

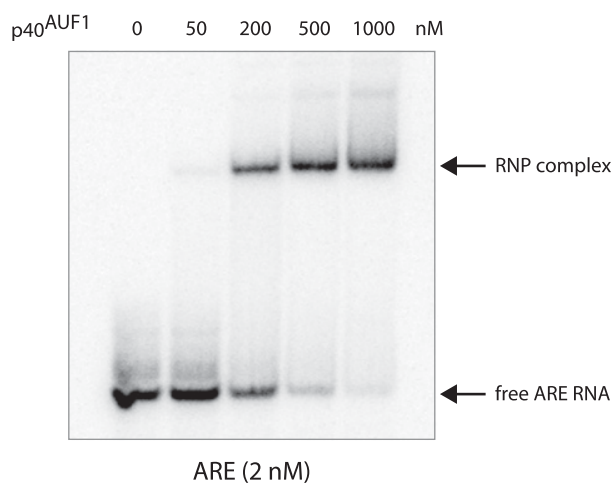

C

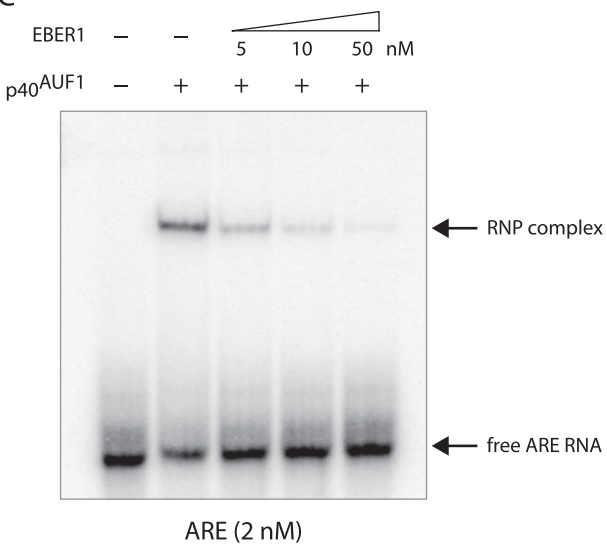

B

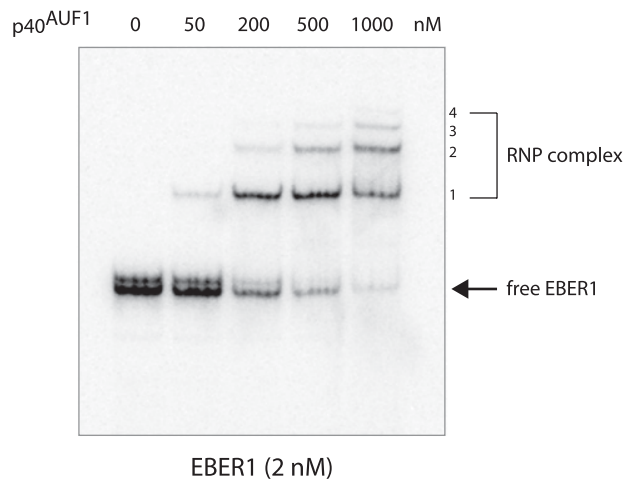

D

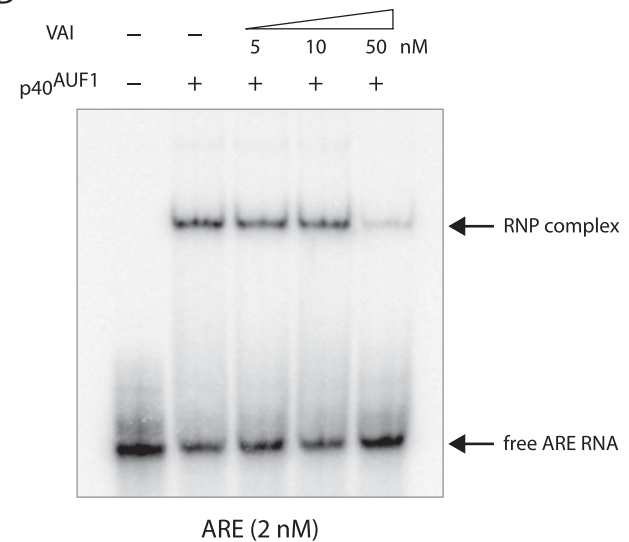

FIGURE 4. EBER1 can compete with the binding of AUF1 to ARE RNA in vitro. $(A, B)$ End-labeled TNF $\alpha$-ARE RNA $(A)$ or EBER1 $(B)$ was incubated at $2 \mathrm{nM}$ with increasing amounts (from 50 to $1000 \mathrm{nM}$ ) of recombinant p40 ${ }^{\mathrm{AUF}}$. RNP complexes were resolved on a native polyacrylamide gel by electrophoresis for $2 \mathrm{~h}$ in $0.5 \times$ TBE buffer. $(C, D)$ ARE RNA $(2 \mathrm{nM})$ was incubated with $200 \mathrm{nM}$ p $40^{\mathrm{AUF} 1}$ (indicated by + ), together with increasing amounts (from 5 to $50 \mathrm{nM}$ ) of EBER1 $(C)$ or VAI RNA $(D)$ as a competitor. All bandshift assays were conducted in the presence of $0.02 \mathrm{mg} / \mathrm{mL}$ tRNA as a nonspecific competitor.

confirmed interaction with EBER1 in vivo by using UV irradiation followed by stringent coimmunoprecipitation, indicating direct RNA-protein crosslinking. Similar analyses would be necessary to validate other hnRNP proteins as true interaction partners of EBER1.

If EBER1 binds and thus restrains AUF1 from interacting with ARE-containing mRNAs in EBV-infected cells, then the presence of EBER1 should increase the levels of AREcontaining mRNAs. In a recent study, Gregorovic et al. (2011) compared the transcript profiles of lymphoblastoid cell lines that were infected with either wild type or an EBV strain carrying a deletion for EBER1. Forty-one mRNAs were found to be significantly down-regulated in the cells lacking EBER1 compared with cells infected with wild-type virus (Gregorovic et al. 2011). A database search for AREs in the 41 down-regulated mRNAs (using ARED) (Bakheet et al. 2001, 2006) revealed that six of the 41 down-regulated transcripts contain an ARE (CHL1, CRIM1, KCNV1, NTRK2, IFNA6, and FUT4). We estimate the cellular abundance of the p40 isoform of AUF1 to be about $6 \times 10^{6}$ molecules (data not shown), while EBER1 amounts to approximately $10^{6}$ molecules in an EBV-infected cell (Lerner et al. 1981). Since multiple regions of EBER1 appear to interact with $440^{\mathrm{AUF} 1}$ (Fig. 4B), it is conceivable that EBER1 may largely sequester the available $\mathrm{p} 40^{\mathrm{AUF} 1}$ molecules and thereby diminish their interaction with ARE-containing mRNAs in vivo. The fact that only a minor cohort of downregulated mRNAs contained AREs (the TNF $\alpha$ mRNA level, e.g., was not affected by the presence of EBER1) (Supplemental Fig. S5) suggests that EBER1 possibly fulfills additional functions other than regulating the stability of AREcontaining mRNAs.

Recently, a novel function of AUF1 in suppression of senescence and telomere maintenance was reported (Pont et al. 2012). AUF1 was shown to bind to the promoter and thereby activate transcription of the telomerase catalytic subunit TERT (telomerase reverse transcriptase). Consistent with our observation that EBER1 can sequester AUF1, 
we found that the mRNA level of TERT is reduced over twofold in EBER1-containing BJAB-B1 cells compared with isogenic BJAB cells (Supplemental Fig. S6). However, it was the p42 and p45 isoforms of AUF1 that were reported to be responsible for activation of TERT gene expression (Pont et al. 2012), whereas our in vivo interaction assay showed that these isoforms of AUF1 interact with EBER1 less well than the p40 isoform. Future studies, optimally in primary lymphocytes rather than immortalized cell lines, will be needed to resolve how interaction of the EBER1 RNP with AUF1 impacts telomere maintenance, ARE-containing mRNA stability, and other aspects of gene expression in EBVassociated lymphomas.

\section{MATERIALS AND METHODS}

\section{EBER1 selection}

$\mathrm{BJAB}$ cells, an EBV-negative Burkitt's lymphoma line, and BJABB1 cells, an EBV-converted BJAB cell line, were used (Menezes et al. 1975; Fresen et al. 1977). For isolating EBER1 with complementary oligonucleotides, a 2 '-O-methyl modified RNA oligonucleotide complementary to EBER1 nucleotides 62-87 was used. The nucleotide at the 3 '-end was not modified to allow oxidization and immobilization on adipic acid dihydrazide Agarose beads (Sigma), as described (Caputi et al. 1999; Borah et al. 2009). Total cell lysate from BJAB-B1 cells was used for isolation of EBER1 with complementary oligonucleotides.

The S1-aptamer (5'-accgaccagaatcatgcaagtgcgtaagatagtcgcgggcc gggg- $\left.3^{\prime}\right)$ or three bacteriophage MS2 coat protein binding sites (5' -cgtacaccatcagggtacgagctagcccatggcgtacaccatcagggtacgactagtaga tctcgtacaccatcagggtacg-3'; MS2-hairpins are underlined) according to the method described by Zhou et al. (2002) were inserted into stem-loop IV of EBER1 (after nucleotide 106) by site-directed mutagenesis using Stratagene's QuikChange SiteDirected Mutagenesis Kit. Aptamer-containing EBER1 constructs were cloned into the lentiviral pTYF vector (Cao et al. 2008) and were expressed from the U6 promoter. Stable BJAB cell lines were generated by lentiviral transduction according to the method previously described (Cao et al. 2008). We lysed $10^{7}$ BJAB cells expressing aptamer-containing EBER1 in $500 \mu \mathrm{L}$ lysis buffer (10 mM Hepes at pH 7.4, $150 \mathrm{mM} \mathrm{KCl,} 3 \mathrm{mM} \mathrm{MgCl}, 2 \mathrm{mM}$ DTT, $0.5 \%$ NP-40, $10 \%$ glycerol) in the presence of protease inhibitor $(1 \mathrm{mM}$ PMSF; $1 \times$ Protease Inhibitor Cocktail Set I; Calbiochem) and RNase inhibitor (80 units of RNasin; Promega). For MS2-mediated selection, $2 \mu \mathrm{g}$ of bacterially purified MS2-MBP fusion protein was added to the lysate and incubated for $3 \mathrm{~h}$ at $4^{\circ} \mathrm{C}$ before addition of amylose resin (NEB). The beads were washed five times with wash buffer $(20 \mathrm{mM}$ Tris at $\mathrm{pH} 7.4$, $200 \mathrm{mM} \mathrm{NaCl}, 2 \mathrm{mM} \mathrm{MgCl} 2,1 \mathrm{mM} \mathrm{DTT}$ ) and eluted in wash buffer containing $10 \mathrm{mM}$ maltose. SDS loading buffer was added to the eluate for Western blot analysis; for Northern blots, RNA was isolated by adding TRIZOL (Invitrogen) to the eluate. Sequences of DNA oligonucleotides that were $5^{\prime}$-end-labeled with $\left[\gamma^{32} \mathrm{P}\right]$ ATP for Northern blot analysis were $5^{\prime}$-cagctggtacttg accgaagac- $3^{\prime}$ (complementary to nucleotides 130-151 of EBER1) and $5^{\prime}$-attagagaatcctgacttgcaaatgctct-3' (complementary to nucleotides 106-134 of EBER2).

\section{SILAC sample preparation and mass spectrometry analysis}

Cells were cultured in RPMI-1640 media formulated for SILAC experiments (Pierce). BJAB cells stably expressing EBER1 or EBER1-MS2 hairpins were cultured in RPMI medium containing either naturally abundant ${ }^{12} \mathrm{C}$-, ${ }^{14} \mathrm{~N}$-arginine and lysine (light medium) or heavy isotope-containing medium $\left({ }^{13} \mathrm{C}-,{ }^{15} \mathrm{~N}\right.$-arginine and lysine) for at least 10 doublings to ensure complete (>95\%) endogenous labeling. We lysed $5 \times 10^{7}$ cells from each sample; equal amounts of proteins as determined by Bradford assay were mixed and subjected to MS2-mediated selection as described above. The eluted proteins were separated by SDSPAGE, stained with colloidal Coomassie, and excised in five gel slices (for the MS2-selection where EBER1-MS2 expressing cells were cultured in heavy medium) or in seven gel slices (for the MS2-selection with reversed labeling). Proteins were prepared for mass spectrometry by cysteine reduction and alkylation, followed by overnight trypsin digestion at $37^{\circ} \mathrm{C}$ (Shevchenko et al. 2006). The tryptic peptides were desalted with $\mathrm{C} 18$ stage-tips (Agilent Technologies) and sequenced in a tandem mass spectrometer (LTQ-Orbitrap) at the Keck Proteomics Facility (Yale University).

The LTQ-Orbitrap raw data were analyzed using the software MaxQuant-Andromeda version 1.1.1.25 to identify the sequenced proteins and to determine their SILAC ratios (Cox et al. 2011). The SILAC label values were set to Arg0/Lys0 and Arg10/Lys6. Proteins with a single SILAC count were included in the quantification. MS/MS spectra were searched against the human International Protein Index (IPI) database (version 3.68). A falsediscovery rate of $1 \%$ was selected using a randomized version of the human protein database. Other applied parameters were "filter labeled amino acids," "re-quantify," and "keep low scoring versions of identified peptides."

\section{Antibodies for Western blot analysis}

Antibodies used for Western blot analyses were as follows: mouse anti-AUF1 at 1:2000 dilution (kind gift of Dr. Gideon Dreyfuss) (Pinol-Roma et al. 1988), rabbit anti-L22 at 1:5000 (Toczyski and Steitz 1993), mouse anti-La (1:500; kind gift of Dr. Ger Pruijn), mouse anti-Flag (1:2000; M2, Sigma), mouse anti-Myc (1:1000; 9E10, Santa Cruz), and mouse anti-nucleolin (1:2000; 4E2, Abcam).

\section{In vivo UV crosslinking and immunoprecipitation}

We washed $10^{7}$ HEK293T or Raji cells with cold PBS, which were resuspended in $2.5 \mathrm{~mL}$ PBS and irradiated in a $10-\mathrm{cm}$ dish on ice with 254-nm UV light at $800 \mathrm{~mJ} / \mathrm{cm}^{2}$. Cells were collected and lysed in $200 \mu \mathrm{L}$ of lysis buffer $(50 \mathrm{mM}$ Tris at $\mathrm{pH} 8.0,1 \mathrm{mM}$ EDTA, $1 \mathrm{mM} \mathrm{DTT}, 0.5 \%$ SDS) followed by heating for $5 \mathrm{~min}$ at $95^{\circ} \mathrm{C}$ and a short sonication pulse to increase solubility. Eight hundred microliters of correction buffer $(62.5 \mathrm{mM}$ Tris at $\mathrm{pH} 8.0$, $187.5 \mathrm{mM} \mathrm{NaCl}, 1.75 \mathrm{mM}$ EDTA, $1.25 \% \mathrm{NP}-40,0.625 \%$ sodium deoxycholate, $12.5 \%$ glycerol) was added together with protease inhibitor $(1 \mathrm{mM}$ PMSF; $1 \times$ Protease Inhibitor Cocktail Set I; Calbiochem) and RNase inhibitor ( 80 units of RNasin; Promega). The lysate was precleared with protein A-Agarose beads for $1 \mathrm{~h}$ at $4^{\circ} \mathrm{C}$, and antibodies (anti-L22 [Toczyski and Steitz 1993], a precipitating anti-AUF1 antibody [Dempsey et al. 1998]) were added 
together with protein A beads to coimmunoprecipitate crosslinked RNAs. After $4 \mathrm{~h}$ of nutation at $4^{\circ} \mathrm{C}$, beads were washed five times with NET-2 buffer (50 mM Tris at $\mathrm{pH} 7.4,150 \mathrm{mM} \mathrm{NaCl}, 0.05 \%$ NP-40). After the final wash step, $100 \mu \mathrm{L}$ TE buffer containing $0.5 \%$ SDS and $200 \mu \mathrm{g}$ Proteinase $\mathrm{K}$ was added to each sample to free RNAs from crosslinked proteins and incubated for $1 \mathrm{~h}$ at $37^{\circ} \mathrm{C}$ before RNAs were extracted using TRIZOL for Northern blot analysis.

HEK293T cells were transfected with the EBER1 expression plasmid pCEP4-EBER1 (EBER1 was cloned into the pCEP4 vector [Invitrogen] and expressed from the U6 promoter) together with one of the AUF1 isoform expressing plasmids (pFlag-CMV2AUF1; kind gift of Dr. Robert Schneider) (Lu et al. 2006) $48 \mathrm{~h}$ prior to irradiation using Effectene (Qiagen). Expression plasmids for Myc-tagged AUF1 isoforms were generated by subcloning AUF1 cDNAs from pFlag-CMV2 vectors into pCEP4 and adding an N-terminal Myc-tag by PCR. For UV crosslinking and competition experiments, HEK293T cells were transfected with pCEP4EBER1, pFlag-CMV2-p40 AUF1, and either pCEP4, pCEP4-Myc$\mathrm{p} 40^{\mathrm{AUF} 1}$, or pCEP4-Myc-p $45^{\mathrm{AUF} 1}$ at a molar ratio of $4: 1: 10$.

\section{Electrophoretic mobility shift assays}

The 38-nt ARE from the TNF $\alpha$ mRNA 3' UTR (5'-gugauuauuua uuauuuauuuauuauuuauuuauuuag- $\left.3^{\prime}\right)$ was used in bandshift assays according to the method previously described (Zucconi et al. 2010). ARE, EBER1, and VAI RNAs were in vitro transcribed by T7 polymerase and gel-purified from polyacrylamide gels according to the method previously described (Fok et al. 2006b). Purified RNAs were alkaline-phosphatase-treated to remove the $5^{\prime}$-phosphate, followed by end-labeling with $\left[\gamma^{-}{ }^{32} \mathrm{P}\right]$ ATP using T4 polynucleotide kinase. Recombinant His-tagged $\mathrm{p} 40^{\mathrm{AUF} 1}$ was purified from Escherichia coli using the expression plasmid pET23-p40 ${ }^{\mathrm{AUF} 1}$ (kind gift of Dr. Robert Schneider) (Lu et al. 2006) and Talon Metal Affinity Resin (Clontech) according to the manufacturer's instructions. For each bandshift reaction, $2 \mathrm{nM}$ labeled RNA was heated at $95^{\circ} \mathrm{C}$ for 3 min prior to incubation on ice for $30 \mathrm{~min}$ with indicated amounts of $\mathrm{p} 40^{\mathrm{AUF1}}$ in a final volume of $10 \mu \mathrm{L}$ containing $10 \mathrm{mM}$ Tris ( $\mathrm{pH} 7.4), 50 \mathrm{mM} \mathrm{NaCl}, 0.5 \mathrm{mM}$ DTT, $0.1 \mathrm{mM} \mathrm{ZnSO}, 1 \mathrm{mM} \mathrm{MgCl}$, $4 \%$ glycerol, $0.2 \mu \mathrm{g}$ tRNA. RNP complexes were resolved on a $6 \%$ nondenaturing polyacrylamide gel in $0.5 \times \mathrm{TBE}$ buffer at $200 \mathrm{~V}$ for $2 \mathrm{~h}$ at $4^{\circ} \mathrm{C}$. Gels were dried and exposed to a phosphor imaging screen.

\section{AUF1 knockdown cell line}

To generate inducible AUF1 knockdown cells, a short-hairpin RNA construct targeting nucleotides 650-670 of AUF1 (NM_031370) (Lal et al. 2004) was cloned into the pTRIPZ vector (Addgene) according to the method previously described (Paddison et al. 2004). The following primer was used to clone the inducible shorthairpin RNA construct: 5 '-tgctgttgacagtgagcgcaGTTGTAGACTGCA CTCTGAtagtgaagccacagatgtaTCAGAGTGCAGTCTACAACtttgcc tactgcctcggatgcctactgcctcgga-3' (the loop region is depicted in italicized lowercase letters; the sequences of resulting siRNAs are underlined). Stable cell lines were generated by lentiviral transduction. Knockdown was induced by the addition of doxycycline at a final concentration of $1 \mu \mathrm{g} / \mathrm{mL}$, and cells were harvested after $5 \mathrm{~d}$.

\section{SUPPLEMENTAL MATERIAL}

Supplemental material is available for this article.

\section{ACKNOWLEDGMENTS}

We thank Dr. Gideon Dreyfuss (University of Pennsylvania) for AUF1 antibodies; Adam Pont and Dr. Robert Schneider (New York University) for AUF1 expression plasmids; Drs. Sumit Borah, Kathryn Gardner, Kasandra Riley, and Kazio Tycowski for helpful comments; and Demian Cazalla for reagents. This work was supported by grant CA16038 from the NIH. J.A.S. is an investigator of the Howard Hughes Medical Institute.

Received June 12, 2012; accepted August 2, 2012.

\section{REFERENCES}

Arao Y, Kuriyama R, Kayama F, Kato S. 2000. A nuclear matrixassociated factor, SAF-B, interacts with specific isoforms of AUF1/ hnRNP D. Arch Biochem Biophys 380: 228-236.

Bakheet T, Frevel M, Williams BR, Greer W, Khabar KS. 2001. ARED: Human AU-rich element-containing mRNA database reveals an unexpectedly diverse functional repertoire of encoded proteins. Nucleic Acids Res 29: 246-254.

Bakheet T, Williams BR, Khabar KS. 2006. ARED 3.0: The large and diverse AU-rich transcriptome. Nucleic Acids Res 34: D111D114.

Blagoev B, Kratchmarova I, Ong SE, Nielsen M, Foster LJ, Mann M. 2003. A proteomics strategy to elucidate functional proteinprotein interactions applied to EGF signaling. Nat Biotechnol 21: 315-318.

Borah S, Wong AC, Steitz JA. 2009. Drosophila hnRNP A1 homologs Hrp36/Hrp38 enhance U2-type versus U12-type splicing to regulate alternative splicing of the prospero twintron. Proc Natl Acad Sci 106: 2577-2582.

Borah S, Darricarrere N, Darnell A, Myoung J, Steitz JA. 2011. A viral nuclear noncoding RNA binds re-localized poly(A) binding protein and is required for late KSHV gene expression. PLoS Pathog 7: e1002300. doi: 10.1371/journal.ppat.1002300.

Cao R, Wang H, He J, Erdjument-Bromage H, Tempst P, Zhang Y. 2008. Role of hPHF1 in H3K27 methylation and Hox gene silencing. Mol Cell Biol 28: 1862-1872.

Caput D, Beutler B, Hartog K, Thayer R, Brown-Shimer S, Cerami A. 1986. Identification of a common nucleotide sequence in the $3^{\prime}$ untranslated region of mRNA molecules specifying inflammatory mediators. Proc Natl Acad Sci 83: 1670-1674.

Caputi M, Mayeda A, Krainer AR, Zahler AM. 1999. hnRNP A/B proteins are required for inhibition of HIV-1 pre-mRNA splicing. EMBO J 18: 4060-4067.

Chen CY, Shyu AB. 1995. AU-rich elements: Characterization and importance in mRNA degradation. Trends Biochem Sci 20: 465470.

Cook HL, Mischo HE, Steitz JA. 2004. The Herpesvirus saimiri small nuclear RNAs recruit AU-rich element-binding proteins but do not alter host AU-rich element-containing mRNA levels in virally transformed T cells. Mol Cell Biol 24: 4522-4533.

Cox J, Neuhauser N, Michalski A, Scheltema RA, Olsen JV, Mann M. 2011. Andromeda: A peptide search engine integrated into the MaxQuant environment. J Proteome Res 10: 1794-1805.

Dempsey LA, Hanakahi LA, Maizels N. 1998. A specific isoform of hnRNP D interacts with DNA in the LR1 heterodimer: Canonical RNA binding motifs in a sequence-specific duplex DNA binding protein. J Biol Chem 273: 29224-29229. 
Drexler HG, Minowada J. 1998. History and classification of human leukemia-lymphoma cell lines. Leuk Lymphoma 31: 305316.

Fok V, Friend K, Steitz JA. 2006a. Epstein-Barr virus noncoding RNAs are confined to the nucleus, whereas their partner, the human La protein, undergoes nucleocytoplasmic shuttling. J Cell Biol 173: 319-325.

Fok V, Mitton-Fry RM, Grech A, Steitz JA. 2006b. Multiple domains of EBER 1, an Epstein-Barr virus noncoding RNA, recruit human ribosomal protein L22. RNA 12: 872-882.

Fresen KO, Merkt B, Bornkamm GW, Hausen H. 1977. Heterogeneity of Epstein-Barr virus originating from P3HR-1 cells. I. Studies on EBNA induction. Int J Cancer 19: 317-323.

Glickman JN, Howe JG, Steitz JA. 1988. Structural analyses of EBER1 and EBER2 ribonucleoprotein particles present in Epstein-Barr virus-infected cells. J Virol 62: 902-911.

Gouble A, Grazide S, Meggetto F, Mercier P, Delsol G, Morello D. 2002. A new player in oncogenesis: AUF1/hnRNPD overexpression leads to tumorigenesis in transgenic mice. Cancer Res 62: 14891495.

Gregorovic G, Bosshard R, Karstegl CE, White RE, Pattle S, Chiang AK, Dittrich-Breiholz O, Kracht M, Russ R, Farrell PJ. 2011. Cellular gene expression that correlates with EBER expression in Epstein-Barr virus-infected lymphoblastoid cell lines. J Virol 85: 3535-3545.

Houmani JL, Davis CI, Ruf IK. 2009. Growth-promoting properties of Epstein-Barr virus EBER-1 RNA correlate with ribosomal protein L22 binding. J Virol 83: 9844-9853.

Iwakiri D, Eizuru Y, Tokunaga M, Takada K. 2003. Autocrine growth of Epstein-Barr virus-positive gastric carcinoma cells mediated by an Epstein-Barr virus-encoded small RNA. Cancer Res 63: 70627067.

Kedar VP, Zucconi BE, Wilson GM, Blackshear PJ. 2012. Direct binding of specific AUF1 isoforms to tandem zinc finger domains of tristetraprolin (TTP) family proteins. J Biol Chem 287: 54595471.

Kitagawa N, Goto M, Kurozumi K, Maruo S, Fukayama M, Naoe T, Yasukawa M, Hino K, Suzuki T, Todo S, et al. 2000. Epstein-Barr virus-encoded poly(A) ${ }^{-}$RNA supports Burkitt's lymphoma growth through interleukin-10 induction. EMBO J 19: 67426750.

Komano J, Maruo S, Kurozumi K, Oda T, Takada K. 1999. Oncogenic role of Epstein-Barr virus-encoded RNAs in Burkitt's lymphoma cell line Akata. J Virol 73: 9827-9831.

Lal A, Mazan-Mamczarz K, Kawai T, Yang X, Martindale JL, Gorospe M. 2004. Concurrent versus individual binding of $\mathrm{HuR}$ and AUF1 to common labile target mRNAs. EMBO J 23: 3092-3102.

Lerner MR, Andrews NC, Miller G, Steitz JA. 1981. Two small RNAs encoded by Epstein-Barr virus and complexed with protein are precipitated by antibodies from patients with systemic lupus erythematosus. Proc Natl Acad Sci 78: 805-809.

Loflin P, Chen CY, Shyu AB. 1999. Unraveling a cytoplasmic role for hnRNP D in the in vivo mRNA destabilization directed by the AUrich element. Genes Dev 13: 1884-1897.

Lu JY, Bergman N, Sadri N, Schneider RJ. 2006. Assembly of AUF1 with eIF4G-poly(A) binding protein complex suggests a translation function in AU-rich mRNA decay. RNA 12: 883-893.

Mann M. 2006. Functional and quantitative proteomics using SILAC. Nat Rev Mol Cell Biol 7: 952-958.

Menezes J, Leibold W, Klein G, Clements G. 1975. Establishment and characterization of an Epstein-Barr virus (EBC)-negative lymphoblastoid B cell line (BJA-B) from an exceptional, EBVgenome-negative African Burkitt's lymphoma. Biomedicine 22: 276-284.

Paddison PJ, Cleary M, Silva JM, Chang K, Sheth N, Sachidanandam R, Hannon GJ. 2004. Cloning of short hairpin RNAs for gene knockdown in mammalian cells. Nat Methods 1: 163-167.
Pinol-Roma S, Choi YD, Matunis MJ, Dreyfuss G. 1988. Immunopurification of heterogeneous nuclear ribonucleoprotein particles reveals an assortment of RNA-binding proteins. Genes Dev 2: 215227.

Pont AR, Sadri N, Hsiao SJ, Smith S, Schneider RJ. 2012. mRNA decay factor AUF1 maintains normal aging, telomere maintenance, and suppression of senescence by activation of telomerase transcription. Mol Cell 47: 5-15.

Raineri I, Wegmueller D, Gross B, Certa U, Moroni C. 2004. Roles of AUF1 isoforms, HuR and BRF1 in ARE-dependent mRNA turnover studied by RNA interference. Nucleic Acids Res 32: $1279-1288$.

Repellin CE, Tsimbouri PM, Philbey AW, Wilson JB. 2010. Lymphoid hyperplasia and lymphoma in transgenic mice expressing the small non-coding RNA, EBER1 of Epstein-Barr virus. PLOS ONE 5: e9092. doi: 10.1371/journal.pone.0009092.

Rosa MD, Gottlieb E, Lerner MR, Steitz JA. 1981. Striking similarities are exhibited by two small Epstein-Barr virus-encoded ribonucleic acids and the adenovirus-associated ribonucleic acids VAI and VAII. Mol Cell Biol 1: 785-796.

Ruf IK, Rhyne PW, Yang C, Cleveland JL, Sample JT. 2000. EpsteinBarr virus small RNAs potentiate tumorigenicity of Burkitt lymphoma cells independently of an effect on apoptosis. J Virol 74: 10223-10228.

Sarkar B, Lu JY, Schneider RJ. 2003. Nuclear import and export functions in the different isoforms of the AUF1/heterogeneous nuclear ribonucleoprotein protein family. J Biol Chem 278: 2070020707.

Shevchenko A, Tomas H, Havlis J, Olsen JV, Mann M. 2006. In-gel digestion for mass spectrometric characterization of proteins and proteomes. Nat Protoc 1: 2856-2860.

Simon MD, Wang CI, Kharchenko PV, West JA, Chapman BA, Alekseyenko AA, Borowsky ML, Kuroda MI, Kingston RE. 2011. The genomic binding sites of a noncoding RNA. Proc Natl Acad Sci 108: 20497-20502.

Srisawat C, Engelke DR. 2002. RNA affinity tags for purification of RNAs and ribonucleoprotein complexes. Methods 26: $156-161$.

Steitz J, Borah S, Cazalla D, Fok V, Lytle R, Mitton-Fry R, Riley K, Samji T. 2011. Noncoding RNPs of viral origin. Cold Spring Harb Perspect Biol 3: pii.a005165. doi: 10.1101/cshperspect. a005165.

Swaminathan S, Tomkinson B, Kieff E. 1991. Recombinant EpsteinBarr virus with small RNA (EBER) genes deleted transforms lymphocytes and replicates in vitro. Proc Natl Acad Sci 88: 15461550.

Toczyski DP, Steitz JA. 1991. EAP, a highly conserved cellular protein associated with Epstein-Barr virus small RNAs (EBERs). EMBO J 10: $459-466$.

Toczyski DP, Steitz JA. 1993. The cellular RNA-binding protein EAP recognizes a conserved stem-loop in the Epstein-Barr virus small RNA EBER 1. Mol Cell Biol 13: 703-710.

Toczyski DP, Matera AG, Ward DC, Steitz JA. 1994. The Epstein-Barr virus (EBV) small RNA EBER1 binds and relocalizes ribosomal protein L22 in EBV-infected human B lymphocytes. Proc Natl Acad Sci 91: 3463-3467.

Wagner BJ, DeMaria CT, Sun Y, Wilson GM, Brewer G. 1998. Structure and genomic organization of the human AUF1 gene: Alternative pre-mRNA splicing generates four protein isoforms. Genomics 48: 195-202.

Wassarman DA, Steitz JA. 1991. Structural analyses of the 7SK ribonucleoprotein (RNP), the most abundant human small RNP of unknown function. Mol Cell Biol 11: 34323445.

Wolin SL, Cedervall T. 2002. The La protein. Annu Rev Biochem 71: 375-403.

Xu N, Chen CY, Shyu AB. 2001. Versatile role for hnRNP D isoforms in the differential regulation of cytoplasmic mRNA turnover. Mol Cell Biol 21: 6960-6971. 


\section{Lee et al.}

Yajima M, Kanda T, Takada K. 2005. Critical role of Epstein-Barr virus (EBV)-encoded RNA in efficient EBV-induced B-lymphocyte growth transformation. J Virol 79: 4298-4307.

Yang L, Aozasa K, Oshimi K, Takada K. 2004. Epstein-Barr virus (EBV)-encoded RNA promotes growth of EBV-infected T cells through interleukin-9 induction. Cancer Res 64: 5332-5337.

Young LS, Rickinson AB. 2004. Epstein-Barr virus: 40 years on. Nat Rev Cancer 4: 757-768.
Zhou Z, Sim J, Griffith J, Reed R. 2002. Purification and electron microscopic visualization of functional human spliceosomes. Proc Natl Acad Sci 99: 12203-12207.

Zucconi BE, Ballin JD, Brewer BY, Ross CR, Huang J, Toth EA, Wilson GM. 2010. Alternatively expressed domains of AU-rich element RNA-binding protein 1 (AUF1) regulate RNA-binding affinity, RNA-induced protein oligomerization, and the local conformation of bound RNA ligands. J Biol Chem 285: 39127-39139. 

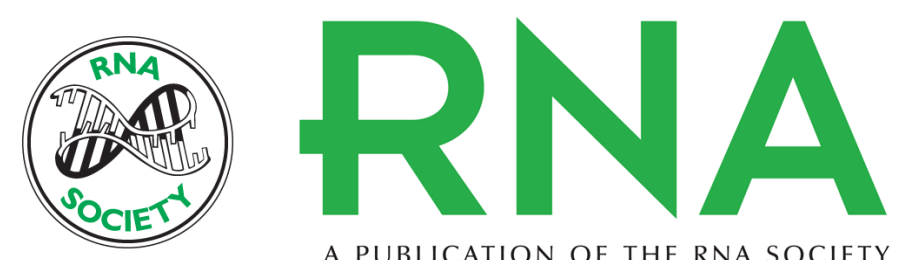

A PUBLICATION OF THE RNA SOCIETY

\title{
AUF1/hnRNP $D$ is a novel protein partner of the EBER1 noncoding RNA of Epstein-Barr virus
}

\author{
Nara Lee, Genaro Pimienta and Joan A. Steitz
}

RNA 2012 18: 2073-2082 originally published online September 25, 2012

Access the most recent version at doi:10.1261/rna.034900.112

\section{Supplemental http://rnajournal.cshlp.org/content/suppl/2012/09/12/rna.034900.112.DC1 Material}

References This article cites 56 articles, 34 of which can be accessed free at: http://rnajournal.cshlp.org/content/18/11/2073.full.html\#ref-list-1

Open Access Freely available online through the RNA Open Access option.

License Freely available online through the RNA Open Access option.

Email Alerting Receive free email alerts when new articles cite this article - sign up in the box at the Service top right corner of the article or click here.

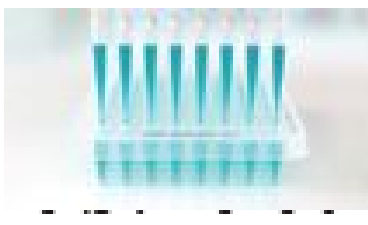

Providing Precise Solutions for your research.

To subscribe to RNA go to:

http://rnajournal.cshlp.org/subscriptions 